\title{
An Experimental Study of Plasma Sheath Effects on Äntennas
}

\author{
G. Tyras, P. C. Bargeliotes, J. M. Hamm, and R. R. Schell \\ Department of Electrical Engineering, The University of Arizona, Tucson, Ariz.
}

(Received November 13, 1964; revised January 18, 1965)

\begin{abstract}
A plasma simulation technique has been developed which can be used to study the effects of homogeneous or inhomogeneous plasma sheath on the radiation pattern deterioration and input impedance of microwave antennas. A tank has been designed and constructed for use in the simulation technique which can reproduce by means of real dielectric materials the dielectric constant encountered in plasma covered antenna research.

The radiation patterns and the input impedances of an annular slot and a thin and long rectangular slot have been successfully measured in the presence of a simulated lossless, homogeneous, and isotropic plasma layer of varied thickness. Comparison with the available theoretical data indicates a generally good agreement, although some differences exist. In the case of the radiation patterns these differences are attributed to the finite distance between the radiator and the receiving antenna on one hand and the inherent inaccuracy of the saddle point method of integration in certain regions on the other hand.
\end{abstract}

\section{Introduction}

Recent aerospace achievements have motivated a great number of investigations into wave propagation through plasma sheaths. Most of these investigations are of theoretical nature and they use an infinite, isotropic, homogeneous, and uniform plasma slab as a model for reentry environment of a radiating system. In order to experimentally verify the theoretical work and to obtain the results for more realistic radiating system and plasma sheath configurations, a laboratory simulation of a plasma sheath is very desirable. The most recent simulation attempts make use of artificial dielectrics, especially rodded media [Rotman, 1962; Golden and Smith, 1964; Golden, 1964]. These attempts are based on the fact that rodded media have refractive indices less than unity under certain conditions [Brown, 1953].

The results of these experiments generally match the theory. Certain important points, however, were not found to coincide with the theory. For example, the sharp peaks at the critical angle, $\sin \theta_{c}=n$, were not found as expected when an $E$ plane slot antenna was used [Golden, 1964]. Such discrepancies are possibly due to "higher-order reflections" from the rodded media which result with increasing oblique incidence [Brown, 1953].

It is the purpose of this paper to introduce another method of simulating the plasma sheath. This method will be seen to provide an exact determination of the simulated plasma parameters and a laboratory geometry for which it is a simple matter to accurately adjust and vary the slab thickness.

The simulation method, to be described in detail in a later section, permitted the recording of the field patterns in the region outside of the plasma sheath. The radiators used were an annular slot and a rectangular slot in a ground plane. Field strength and admittance measurements were made for each antenna with various slab thicknesses. 


\section{Theoretical Background}

The problem of a long, narrow rectangular slot clad with a plasma layer attracted the attention of many authors. Newstein and Lurye [1956] first formulated the problem and derived the expression for the radiation field. Omura [1962] carried out numerous calculations of the radiation patterns where he showed the dependence of the radiation field as affected by the thickness of the plasma slab and the plasma index of refraction. Tamir and Oliner [1962] interpreted the results of the radiation field in terms of "leaky waves." Galejs [1964a, b] considered the problem of finding the admittance of rectangular and annular slots covered with a plasma layer.

In the case of a long, narrow rectangular slot with a constant excitation $V$ and covered with a plasma layer of thickness " $a$ " and the equivalent index of refraction " $n$ " the single component of the far zone magnetic field is given by [Newstein and Lurye, 1956; Omura, 1962; Tamir and Oliner, 1962]

$$
H_{x 0}=\frac{-\omega \epsilon_{0} n^{2} V}{\sqrt{2 \pi}} G(\theta) \frac{e^{i\left(k_{0} \rho-\pi / 4\right)}}{\sqrt{k_{0 \rho}}}
$$

where

$$
G(\theta)=\cos \theta\left[n^{2} \cos \theta \cos \left(k_{0} a \sqrt{n^{2}-\sin ^{2} \theta}\right)-i \sqrt{n^{2}-\sin ^{2} \theta} \sin \left(k_{0} a \sqrt{n^{2}-\sin ^{2} \theta}\right)\right]^{-1}
$$

and $\theta$ is the polar angle as shown in figure 1.

In the case of a narrow annular slot of mean radius " $b$ " the corresponding expression for the single component of the far zone magnetic field is found in the appendix to be

$$
H_{\phi 0}=-i \omega \epsilon_{0} n^{2} b V J_{1}\left(k_{0} b \sin \theta\right) G(\theta) \frac{e^{i\left(k_{0} r-\pi / 4\right)}}{r} .
$$

Since the factor $G(\theta)$ appears in both (1) and (3), the radiation patterns for the rectangular and the annular slot will be quite similar when $k_{0} b<1.5$ and $\theta$ is not too close to zero. ${ }^{1}$ Equations (1) and (3) are valid for real as well as complex values of the plasma index of refraction n, i.e., lossless and lossy plasmas.

${ }^{1}$ When $k_{0} b<1.5$ then $J_{1}\left(k_{0} b\right) \sim k_{0} b$.

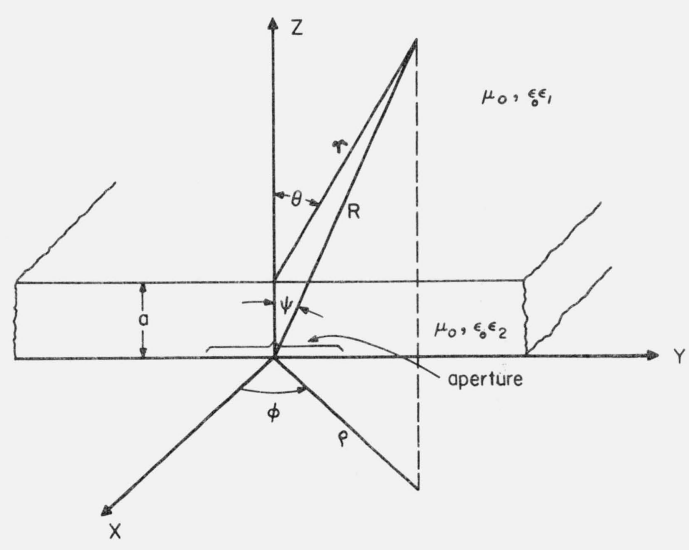

Figure 1. Plasma clad slot antenna geometry. 
FIGURE 2. Theoretical radiation patterns of a plasma clad annular slot.

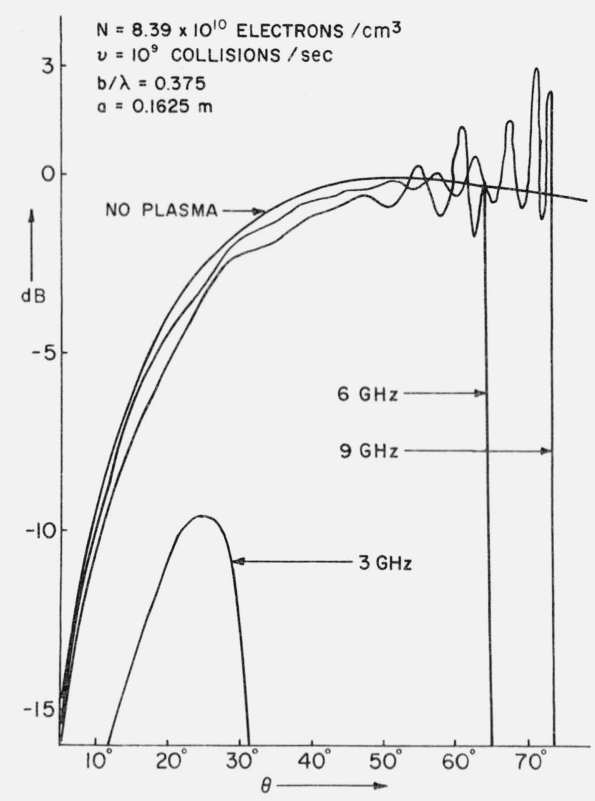

Both the numerical results of Omura [1962] and the "leaky wave" analysis of Tamir and Oliner [1962], as well as the simple consideration of geometric optics, predict that the radiation pattern will have peaks in the neighborhood of the critical angles. Moreover, as the thickness of the layer increases, progressively more minor peaks appear in the radiation pattern while the major lobes are still found in the vicinity of the critical angles [Tamir and Oliner, 1962]. This fact is also apparent in the theoretical radiation patterns of an annular slot shown in figure 2.

The difficulty that arises in the plasma simulation attempts stems from the fact that the real part of the plasma dielectric constant is less than unity, namely, $\epsilon_{p} / \epsilon_{a}<1$ where $\epsilon_{p}$ is the plasma dielectric constant and $\epsilon_{a}$ is the dielectric constant of free space. If, however, $\epsilon_{p} / \epsilon_{a}=\epsilon_{p s} / \epsilon_{a s}<1$ where the subscripts "ps" and "as" denote plasma simulation and air simulation, respectively, it is seen that a simulated plasma environment depends on the ratio of the dielectric constants and not on their absolute values. Thus an artificial plasma environment can be created by covering a radiator or a scatterer under investigation with a medium having a dielectric constant less than that of the free space simulator.

A major requirement of the free space simulator is that it be in the liquid form to allow movement of measuring equipment through it. Furthermore, it should have a low loss tangent to minimize signal attenuation in the medium. The plasma sheath simulator can be any foamy material with $\epsilon_{r} \sim 1$ or simply air itself. Such a combination of dielectric materials will simulate a plasma with $0<\epsilon_{p}<1$.

With such a simulation technique, the plasma parameters can be scaled and properly defined. Consider the rectangular and the annular slabs as examples. If in (1) or (3) we set

$$
\begin{aligned}
n_{s} & =n \\
k_{s} a_{s} & =k_{0} a \\
k_{s} b_{s} & =k_{0} b,
\end{aligned}
$$

where the subscript " $s$ " denotes the equivalent simulated parameters, then it will follow that the ratio $H_{s} / H_{0}$ will be constant for all angles $\theta$. 
The results of (4) can also be obtained directly from Maxwell's equations [Stratton, 1941]. It suffices to require that the two ratios remain invariant

$$
\begin{aligned}
& c_{1}=\mu \epsilon\left(\frac{l}{\tau}\right)^{2} \\
& c_{2}=\mu \sigma \frac{l^{2}}{\tau},
\end{aligned}
$$

where $\tau$ is the characteristic period and $l$ denotes length. In the present case we are dealing with lossless dielectrics hence only $c_{1}$ need to be considered. Now the invariance of $c_{1}$ requires

$$
\mu_{s} \epsilon_{s} l_{s}^{2} \omega_{s}^{2}=\mu \epsilon l^{2} \omega^{2}
$$

which leads directly to the latter two expressions of (4). Moreover, it is to be noted that if the dielectric permittivities are scaled by some factor, say $\kappa$, then this factor must apply to all regions of space. Thus, if $\epsilon_{1}$ refers to the plasma layer and $\epsilon_{2}$ to the outer region then it follows that

$$
\kappa=\frac{\epsilon_{1}}{\epsilon_{1 s}}=\frac{\epsilon_{2}}{\epsilon_{2 s}}
$$

and consequently

$$
\frac{\epsilon_{1}}{\epsilon_{2}}=\frac{\epsilon_{1 s}}{\epsilon_{2 s}}
$$

which is equivalent to first expression of (4).

It should be noted that configurations involving anisotropic plasma layers such as considered by Wait [1961], Hodara and Cohn [1962], and others cannot be simulated by the present technique.

\section{Experimental System and Results}

\subsection{Simulation System}

The study of a table of dielectric materials [Von Hippel, 1958] at $10 \mathrm{GHz}$ has revealed that the requirements of the free space simulating medium, i.e., low loss-tangent, noncorrosiveness, and stability are satisfactorily met by Aroclor 1232. The combination of air and Aroclor $1232\left(\epsilon_{r}=2.78\right.$ and $\tan \delta=0.008$ ) will result in an $n_{s}=0.60$ corresponding to an electron density per cubic centimeter $N=7.83 \times 10^{9} f_{0}^{2}$, where $f_{0}$ is the actual antenna operating frequency in $\mathrm{GHz}$. With the simulating frequency $f_{s}=10 \mathrm{GHz}$, air layer $a_{s}=2.9 \mathrm{~cm}$, slot mean radius $b_{s}=0.675 \mathrm{~cm}$, and the simulating tank containing Aroclor 1232 , it follows that $a / \lambda_{0}=1.612$ and $b / \lambda_{0}=0.375$. As a consequence of the scaling defined in (4) and since the plasma's index of refraction is a function of the wave frequency, the plasma environment that can be represented by this system will depend on the wave frequency chosen. Table 1 shows typical physical conditions of plasma sheath antenna environment that can be simulated with the Aroclor 1232 as free space and air as the plasma layer dielectric for the case of the annular slot.

TABLE 1. Plasma sheath antenna environment representable by the system

\begin{tabular}{c|c|c|c}
\hline \hline$f_{0}$ & $N\left(\right.$ elect./cm $\left.{ }^{3}\right)$ & $a$ & $b$ \\
\hline & & & \\
$\mathrm{GHz}$ & & $\mathrm{cm}$ & $\mathrm{cm}$ \\
3 & $7.02 \times 10^{10}$ & 16.1 & 3.75 \\
6 & $2.80 \times 10^{11}$ & 8.1 & 1.88 \\
9 & $6.32 \times 10^{11}$ & 5.4 & 1.25 \\
12 & $1.13 \times 10^{12}$ & 4.0 & 0.94 \\
15 & $1.75 \times 10^{12}$ & 3.23 & 0.75 \\
\hline
\end{tabular}


FIGURE 3. The simulation tank.

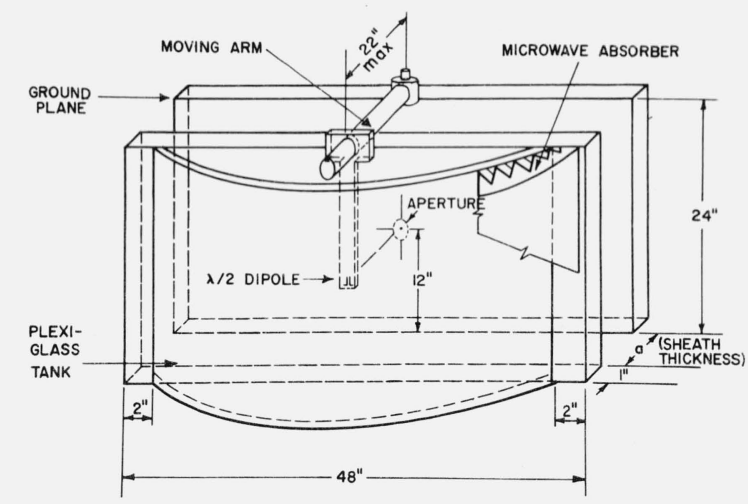

A semicylindrical tank of 22 in. inside radius and $24 \mathrm{in}$. high, as shown in figure 3 , was designed and subsequently built to the design specifications. The wall material is plexiglass, $\epsilon_{r}=2.59$. The flat wall of the tank is made of 1 -in. plexiglass plate and the curved wall of 0.25 -in. plexiglass. The tank holds $78 \mathrm{gal}$. of Aroclor $1232 \mathrm{oil}, \epsilon_{r}=2.78$. At the operating frequency of $10 \mathrm{GHz}$, the tank allows a separation of the transmitting and the receiving antennas by at least 28 wavelengths.

Since the relative dielectric constants of the tank material and the oil are so close in value, the interface between them has negligible effect on the wave propagation. The reflections from the curved plexiglass-air interface were successfully reduced to a desirable level by placing high performance microwave absorber against the outside wall of the tank.

With the simulation tank located on top of a stationary bench, the ground plane could be situated conveniently at any distance in back of the simulation tank thus allowing the simulation of the plasma sheath of arbitrary thickness. The ground plane was mounted in the vertical plane atop a wooden cart equipped with rollers for ease of movement. The gap between the ground plane and the simulation tank was adjusted to the desired dimension by means of spacers and clamps to avoid accidental movement. The effects of the finite dimensions of both the ground plane and the simulated plasma layer were further alleviated by placing high performance microwave absorber around the finite boundaries of the simulated plasma layer. The absorber in this position acts as a matched termination of the plasma layer edges.

The pick-up antenna consisted of a half-wave electric dipole on the end of a RG58A/U coaxial cable. The definition of the plane of the dipole was achieved by embedding the dipole in a plexiglass strip while its feeding cable was taken out of the tank along an L -bracket also made of plexiglass. The L -bracket was rigidly connected to a vertical shaft, the axis of which coincided with the center of the aperture in the ground plane. This type of arrangement allowed approximately $\pm 70^{\circ}$ coverage in the $\psi$ direction for the largest simulated plasma layer of $2 \mathrm{in}$. in accordance with the slot coordinates as shown in figure 1 .

The availability of theoretical results for the radiation patterns of an annular slot and a thin, long rectangular slot motivated the experimental investigation of these two types of radiators. An annular slot was constructed using brass stock because of the ease for precision machining. Since an annular slot of such narrow width has a very small impedance, a matching transformer was necessary to match the slot impedance to the $50-\Omega$ coaxial feed line. A coaxial conical taper of 5.2 wavelengths was machined as an integral part of the slot piece and fulfilled the impedancematching requirements. Nylon with $\epsilon_{r}=3.02$ at $10 \mathrm{GHz}$ served as the dielectric of the coaxial conical taper. The desired slot had a mean radius of 0.256 -in. and 0.020 -in. width. Figure 4 shows the schematic of the slot and tapering section with detailed machining dimensions. The rectangular slot was constructed using two waveguide sections as shown in figure 5 . Two brass pieces were machined and inserted in the guides, with the small dimension of the guide gradually tapering to a 0.0312 -in. slot. 


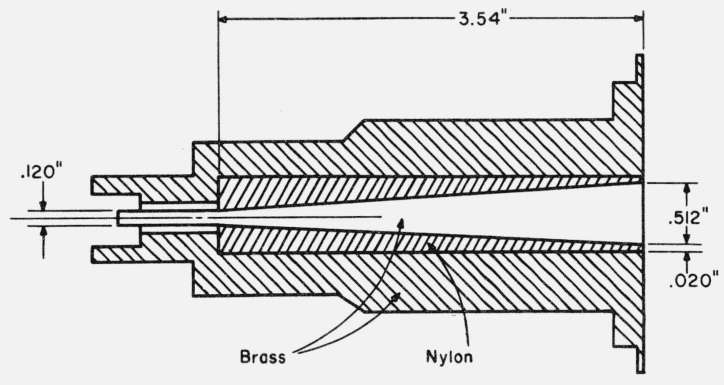

FigURE 4. Annular slot construction.

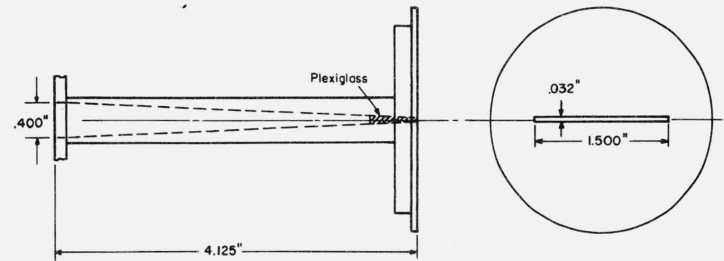

Figure 5. Rectangular slot construction.

Because of the inherent difficulties encountered in the measurement of the slot antenna admittance at the aperture itself, the voltage standing wave ratio (VSWR) and the shift of the null from the short circuit to the loaded condition were measured at the input of the tapered transition section. These two parameters were measured by means of a standard slotted-section technique for different simulated plasma thicknesses. The knowledge of the VSWR and the null shift permits the calculation of the slot admittance by using the Smith Chart or the well-known transmission line equation. The measured value of the admittance could be transferred to any point on the taper had the variation of the characteristic admittance with the taper length been known which is not the case for the linear taper used. A short-circuit test performed on the taper indicates that this structure has negligible losses and, consequently, it can be considered as an ideal transformer. Hence, the functional dependence of the layer thickness on the slot radiation admittance as measured at the input of the taper should be the same as it would be at the plane of the slot itself.

\subsection{Results}

The experimental antenna patterns are shown in figure 6 for the annular slot. Of importance to note is the occurrence of the radiation maxima near the theoretical critical angle, $\theta_{c}=37^{\circ}$ and a low gain region near $\theta=0^{\circ}$. This is entirely as predicted by the theory. Theoretical curves for $a=0.353 \lambda_{0}$ and for $\mathrm{a}=1.41 \lambda_{0}$ have been plotted and are compared with the experimental values in figures $6 a$ and $6 b$, respectively. Figure $6 c$ shows experimental curves for $a=0.706 \lambda_{0}$ and for $a=1.06 \lambda_{0}$. Very good agreement with theory is evident in figure $6 \mathrm{a}$, but significant discrepancies are noted in figure 6b. As will be seen later, the same situation occurred in the case of the rectangular slot.

The results of the admittance measurements for the annular slot are seen in figure 7. Reference to theoretical curves [Galejs, 1964a] shows that the experimental curves are of the same general shapes as those predicted. One notable discrepancy is seen in the curve for the normalized conductance. The theory predicts approximately a $5: 1$ ratio between the maximum and minimum values of $G / Y_{0}$, while the experimental curve shows approximately a 1.1:1 ratio.

The radiation patterns of the rectangular slot are seen in figure 8. Again, note the occurrence of radiation maxima near the predicted critical angle, $\theta_{c}=37^{\circ}$. Theoretical curves for a $=0.353 \lambda_{0}$ and for $a=1.41 \lambda_{0}$ have been plotted and compared with the experimental values in figures $8 \mathrm{a}$ and $8 \mathrm{~b}$ respectively. As observed previously, the pattern for the small sheath thickness of $0.353 \lambda_{0}$ agrees quite well with theory, while the same significant discrepancies are noted for the large thickness. Figure 8c shows experimental radiation patterns for sheath thicknesses of $0.706 \lambda_{0}$ and $1.06 \lambda_{0}$. 

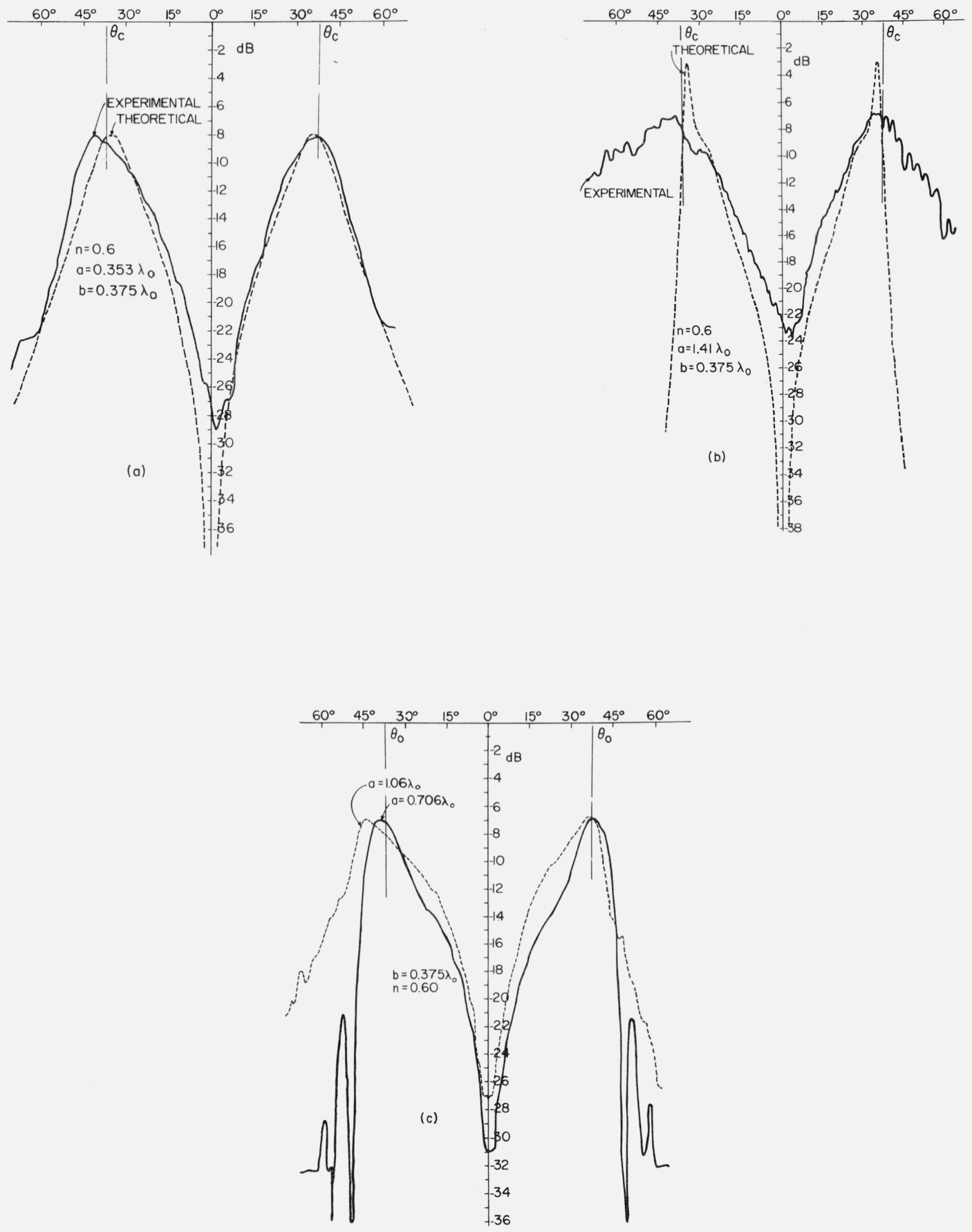

FIGURE 6. Experimental and theoretical radiation patterns of the plasma clad annular slot.

In evaluating these results. it is well to keep in mind any approximations made in obtaining the theoretical radiation patterns. For the problem at hand, the method of saddle point integration has been used. This method allows the representation of an integral in the form of a series of inverse powers of distance from the source [Brekhovskikh, 1960]. It is assumed that a portion of the integrand, which in the present problem is the angular variation of the pattern, varies slowly. That is, its derivatives are sufficiently small so that the expansion may be limited to the first term. This approximation becomes better further from the source. 

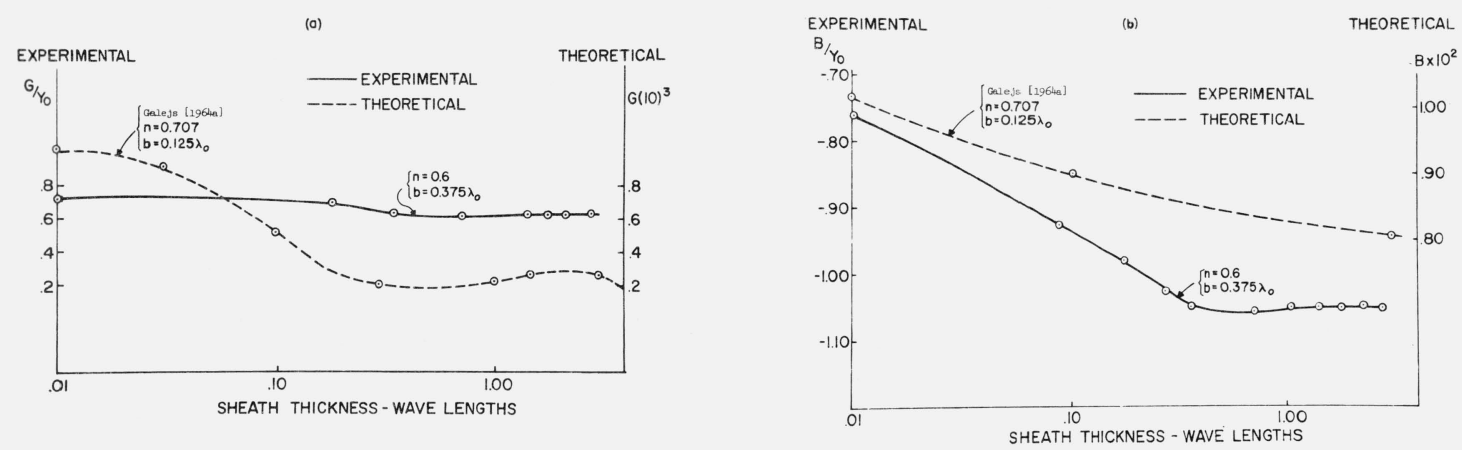

FiguRE 7. Admittance characteristics of the plasma clad annular slot.
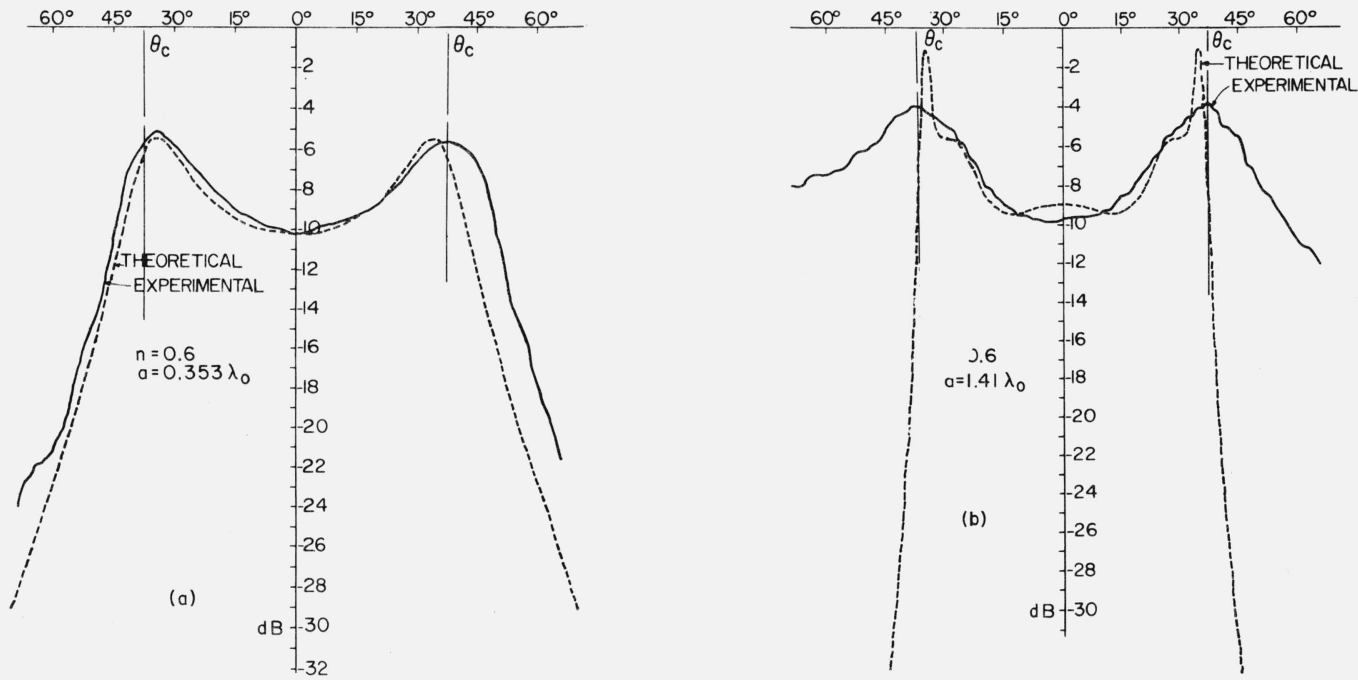

FIGURE 8. Experimental and theoretical radiation patterns of the plasma clad rectangular slot.

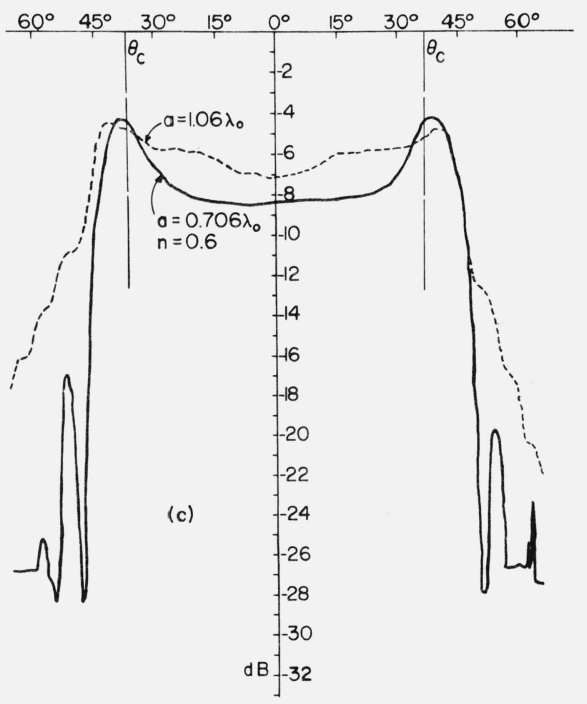


Figure 9. Functional dependence of the minimum farfield distance versus sheath thickness.

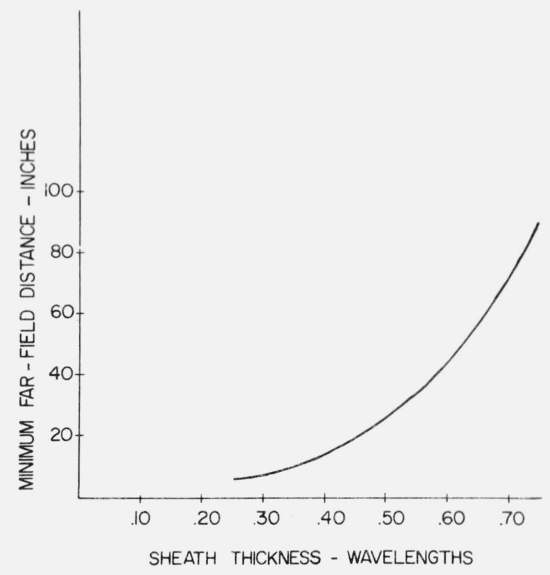

Now, reference to figure 8 shows that for large plasma thicknesses, the angular variation becomes quite rapid near the critical angle. In fact, one can show that at the critical angle, the first derivative is proportional to the square of the layer thickness. The situation, then, is the following. In the case of small plasma thicknesses, the slowly varying assumption is valid for the radial spacing between source and pickup available to the simulation technique. As the thickness increases, however, the angular variation near the critical angle becomes more rapid and the calculated results become less accurate. Physical limitations prevent the increase of radial spacing. Thus, to obtain better agreement with the simulated patterns, it becomes necessary to include a second term in the saddle point approximation. At present, however, the second term has not yet been evaluated and, consequently, the theoretical results include the contribution of the first term only.

Another major factor contributing to the discrepancies in the radiation patterns stems from the fact that the physical boundaries of the system are not sufficient to provide the minimum required far-field distance for thicknesses greater than $0.45 \lambda_{0}$. The dependence of the minimum far-field distance on the sheath thickness is seen in figure 9. This curve was plotted by considering the half power bandwidth of the radiation pattern around the critical angle. The half power bandwidths were calculated from (2). Now from Silver [1949] we have

$$
\theta_{H P B W} \sim 60^{\circ}\left(\frac{\lambda}{d}\right) \min
$$

which yields $d / \lambda$, and subsequently the minimum far-field distance. Thus the radiation pattern for $a=0.353 \lambda_{0}$ is well in the radiation field, while that for $a=1.41 \lambda_{0}$ is in the near field. ${ }^{2}$

The admittance curves for the rectangular slot are seen in figure 10. Theoretical calculations have been made by Galejs [1964b]. Comparison with these curves shows that the experimental curve for the normalized conductance agrees quite well with the available theory when the sheath thickness is larger than $0.5 \lambda_{0}$. The same can be said for the normalized susceptance curve.

\footnotetext{
${ }^{2}$ We are indebted to the reviewer for suggesting this method of determining the minimum far-field distance.
} 

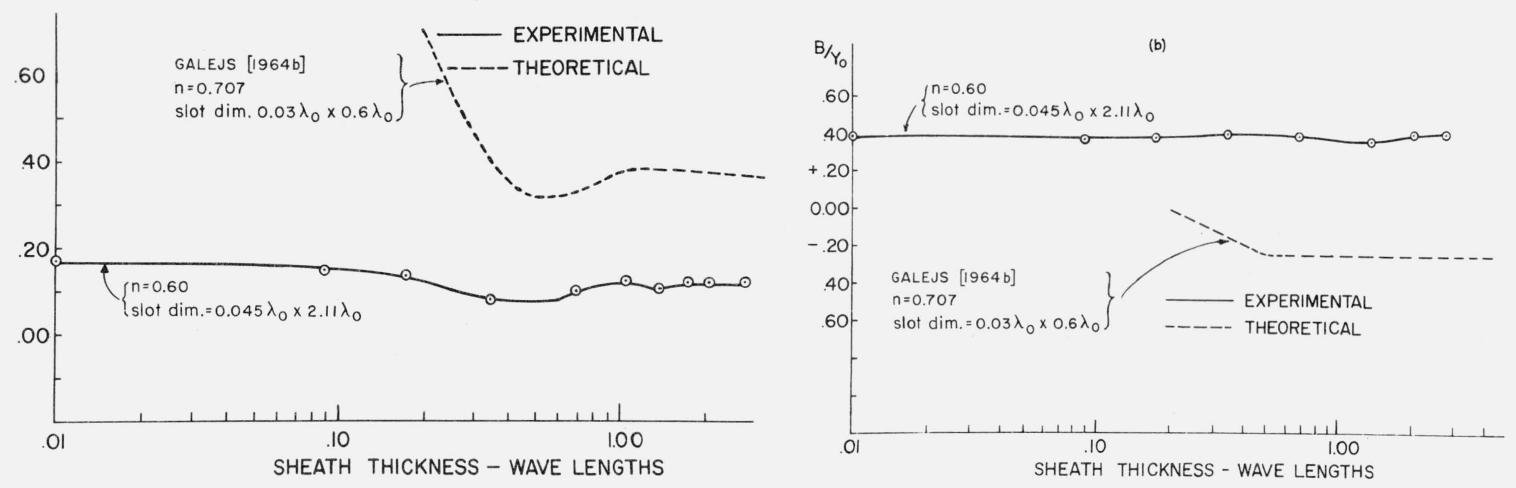

FIGURE 10. Admittance characteristics of the plasma clad rectangular slot.

\section{Conclusions}

In the present work only the simplest case of the plasma sheath environment was treated, i.e., the case of a lossless, isotropic, and homogeneous plasma. The simulation technique has been successfully tested only for this simplest case, but it is clear that it may be used for the study of more complex plasma sheath models. The inherent simplicity of this experimental system and its versatility suggest that the method is capable of simulating more realistic plasma sheath environments which are not readily amenable to analytic solutions.

The most serious limitation of the present technique is the fact that the method is not capable of simulating plasmas characterized by a negative dielectric constant. Moreover, the effective simulated dielectric constant of 0.36 achieved with the Aroclor 1232-air combination cannot be readily lowered because of the lack of suitable liquid dielectrics with $\epsilon_{r}>2.8$. However, since the reentry communication systems can be expected to work well above the plasma frequency, the above limitations may not be serious ones indeed.

This research was sponsored by the Air Force Cambridge Research Laboratories, Office of Aerospace Research, USAF, under the contract AF 19 (628)-3834.

\section{Appendix}

Consider a narrow annular slot of mean radius " $b$ " cut in an infinitely conducting plane of infinite extent and covered with a layer of isotropic and homogeneous plasma of thickness " $a$ " and the index of refraction " $n$ ". A constant voltage " $V$ " is assumed to be present across the slot. The horizontal plane $z=a$ coincides with the interface of the homogeneous plasma layer and the surrounding medium. For convenience, reference will be given to the surrounding air medium (0) and plasma medium (1).

It is clearly seen from the symmetry of the problem that there is no variation in the $\phi$-coordinate, i.e., $\frac{\partial}{\partial \phi}=0$, and only the $H_{\phi}$ component of the magnetic field exists. Furthermore, it is noted that the electric field at the aperture is

$$
E_{\rho}=-V \delta(\rho-b) \delta(z) .
$$


Assuming the $e^{-i \omega t}$ time-dependence and suppressing it throughout, the electric field components are given by

$$
E_{\rho}=\frac{1}{i \omega \epsilon_{0} \epsilon} \frac{\partial H_{\phi}}{\partial z}
$$

and

$$
E_{z}=-\frac{1}{i \omega \epsilon_{0} \epsilon} \frac{1}{\rho} \frac{\partial}{\partial \rho}\left(\rho H_{\phi}\right)
$$

where $H_{\phi}$ satisfies the equation

$$
\frac{\partial}{\partial \rho}\left[\frac{1}{\rho} \frac{\partial}{\partial \rho}\left(\rho H_{\phi}\right)\right]+\frac{\partial^{2} H_{\phi}}{\partial z^{2}}+k^{2} H_{\phi}=\left\{\begin{array}{cc}
-i 2 \omega \epsilon_{0} n^{2} V \delta(\rho-b) \delta(z), & 0<z<a \\
0 & , z>a .
\end{array}\right.
$$

The solution to the problem can be formulated readily using Fourier-Bessel transform [Stratton, 1941]. The single component of the magnetic field in each one of the two regions has the form

$$
\begin{aligned}
& H_{\phi 0}=\frac{i V b \omega \epsilon_{0} n^{2}}{2} \int_{-\infty}^{+\infty} \frac{e^{-\gamma_{0}(z-a)} J_{1}(\lambda b) H_{1}^{(1)}(\lambda \rho) \cdot \lambda d \lambda}{\left[\gamma_{1} \sinh \left(\gamma_{1} a\right)+n^{2} \gamma_{0} \cosh \left(\gamma_{1} a\right)\right]} \\
& H_{\phi 1}=\frac{i V b \omega \epsilon_{0} n^{2}}{2} \int_{-\infty}^{+\infty} \frac{\left[\gamma_{1} \cosh \gamma_{1}(a-z)+n \gamma_{1} \sinh \gamma_{1}(a-z)\right]}{\gamma_{1}\left[\gamma_{2} \sinh \left(\gamma_{1} a\right)+n^{2} \gamma_{0} \cosh \left(\gamma_{1} a\right)\right]} J_{1}(\lambda b) H_{1}^{(1)}(\lambda \rho) \lambda d \lambda,
\end{aligned}
$$

where

$$
\begin{gathered}
\gamma_{i}=\sqrt{\lambda^{2}-k_{i}^{2}} \\
k_{i}=2 \pi / \lambda_{i} .
\end{gathered}
$$

Changing to spherical coordinates in the configuration space and taking $\lambda=k_{0} \sin \beta$ in the transform space, (A4a) is changed into a contour integral in the complex $\beta$-plane. The evaluation of the contour integral by the saddle point method gives the far-zone field in the air region;

$$
H_{\phi 0}=-i \omega \epsilon_{o} n^{2} b V J_{1}\left(k_{0} b \sin \theta\right) G(\theta) \frac{e^{i\left(k_{0} r-\pi / 4\right)}}{r}
$$

where

$$
G(\theta)=\cos \theta\left[n^{2} \cos \theta \cos \left(k_{0} a \sqrt{n^{2}-\sin ^{2} \theta}\right)-i \sqrt{n^{2}-\sin ^{2} \theta} \sin \left(k_{0} a \sqrt{n^{2}-\sin ^{2} \theta}\right)\right]^{-1} .
$$

It is clear that (A5) and (A6) are valid for real as well as complex values of the plasma index of refraction, $n$.

Equation (A5) gives the complete solution of the fields in the radiation zone. However, "leaky wave" poles, poles arising from the singularities of the contour integral and located in the improper Riemann sheet, may significantly affect the near and intermediate zones. These "leaky waves" exhibit a radial attenuation and their effect on the radiation field diminishes even when strongly excited. An extensive treatment of complex poles is given by Tamir and Oliner [1962]. 


\section{References}

Brekhovskikh, L. M. (1960), Waves in layered media, 6, 245-250 (Academic Press, Inc., New York, N.Y.).

Brown, M. A. (May 1953), Artificial dielectrics having refractive indices less than unity, Proc. IEE Monograph No. 62R, 100, pt 4, 51-62.

Galejs, J. (March 1964a), Admittance of annular slot antennas radiating into a plasma layer, Radio Sci. J. Res. NBS 68D, No. 3, 317-324.

Galejs, J. (Nov. 1964b), Slot antenna impedance for plasma layers, IEEE Trans. Ant. Prop. AP-12, No. 6, $738-745$.

Golden, K. E. (May 1964), A study of artificial dielectrics, Plasma Res. Lab. Aerospace Corp., Contract No. AF 04(695)-269.

Golden, K. E., and T. M. Smith (March 1964), Simulation of a thin plasma sheath by a plane of wires, Plasma Res. Lab. Aerospace Corp., Contract No. AF 04(695)-269.

Hodara, H., and G. I. Cohn (Sept. 1962), Radiation from a gyro-plasma coated magnetic line source, IRE Trans. Ant. Prop. AP-10, No. 5, 581-593.

Newstein, M., and J. Lurye (July 1956), The field of a magnetic line source in the presence of a layer of complex refractive index, Technical Res. Group, Sci. Rpt. No. 1, New York, N.Y.

Omura, M. (Dec. 1962), Radiation pattern of a slit in a ground plane covered by a plasma layer, Air Force Cambridge Res. Lab., AFCRL-62-958.

Rotman, W. (Jan. 1962), Plasma simulation by artificial dielectric and parallel-plate media, IRE Trans. Ant. Prop. AP-10, No. 1, 82-95.

Silver, S. (1949), Microwave antenna theory and design (McGraw-Hill Book Co., Inc., New York, N.Y.).

Stratton, J. A. (1941), Electromagnetic theory, 488-489 (McGraw-Hill Book Co., Inc., New York, N.Y.).

Tamir, T., and A. A. Oliner (Jan. 1962), The influence of complex waves on the radiation field of a slot-excited plasma layer, IRE Trans. Ant. Prop. AP-10, No. 1, 55-65.

von Hippel, A. R. (1958), Dielectric materials and applications, 300-370 (John Wiley \& sons, New York, N.Y.).

Wait, J. R. (1961), The electromagnetic fields of a dipole in the presence of a thin plasma sheet, App. Sci. Res. Sec. B, 8, 397-417.

(Paper 69D6-516) 